\title{
COVID-19 patients exhibit less pronounced immune suppression compared with bacterial septic shock patients
}

\author{
Matthijs Kox ${ }^{1,2^{*}}$, Tim Frenzel ${ }^{1,2}$, Jeroen Schouten ${ }^{1,2}$, Frank L. van de Veerdonk ${ }^{2,3}$, Hans J. P. M. Koenen ${ }^{2,4}$, and \\ Peter Pickkers ${ }^{1,2}$, on behalf of the RCI-COVID-19 study group
}

Keywords: HLA-DR, mHLA-DR, COVID, COVID-19, SARS-CoV-2, Monocytes, Immune suppression

Low monocytic (m)HLA-DR expression is the most widely used marker of innate immune suppression in critically ill patients. We recently showed that in bacterial septic shock patients, low mHLA-DR expression is prevalent and associated with the development of secondary infections [1]. At the end of March 2020, there were in excess of 800,000 confirmed cases of coronavirus disease 2019 (COVID-19) worldwide, of whom more than 12,000 from the Netherlands. Several reports suggest that patients with severe COVID-19 may suffer from a hyperinflammatory "cytokine storm" $[2,3]$. However, unlike SARS-CoV infection, high levels of antiinflammatory mediators (e.g. IL-10 and IL-4) have also been reported in COVID-19 [3]. Although there are few indications that secondary infections are common in COVID-19 patients, one study reported that $16 \%$ of COVID-19 patients who died developed secondary infections [4], which might indicate an immune-suppressed state. Herein, we explored mHLA-DR expression kinetics in a cohort of 24 critically ill COVID-19 patients.

Between March 18 and 27, all COVID-19 patients admitted to our intensive care unit (ICU) were in- cluded in this prospective observational study. COVID-19 was confirmed by two positive RT-PCR tests for SARS-CoV-2 in throat swabs and by CT scan findings. Fourteen patients were transferred from other ICUs. The median ICU length of stay at the time of study inclusion was 3 days. The study was carried out in accordance with the applicable rules concerning the review of research ethics committees and informed consent in the Netherlands. All patients or legal representatives were informed about the study details and allowed to abstain from participation. Ethylenediaminetetraacetic acid (EDTA)-anticoagulated blood was stored at $4-8^{\circ} \mathrm{C}$ until mHLA-DR expression analysis (performed within 2 $\mathrm{h}$ after withdrawal). Expression levels were determined using the Anti-HLA-DR/Anti-Monocyte Quantibrite assay (BD Biosciences, San Jose, USA) on a Navios flow cytometer and software (Beckman Coulter, Brea, USA). Total number of antibodies bound per cell ( $\mathrm{mAb} / \mathrm{cell})$ were quantified using a standard curve constructed with Quantibrite phycoerythrin beads (BD Biosciences). All other data were extracted from the electronic patient record. For patients who were transferred from other ICUs,

\footnotetext{
* Correspondence: Matthijs.Kox@radboudumc.nl

'Department of Intensive Care Medicine, Radboud University Medical Center, Internal Mail 710, PO Box 9101, 6500 HB Nijmegen, The Netherlands

${ }^{2}$ Radboud Center for Infectious Diseases (RCI), Radboud University Medical Center, Nijmegen, The Netherlands

Full list of author information is available at the end of the article
} 
Table 1 Patient characteristics

Characteristics
Age, years
Sex
Female
Male
Body mass index, $\mathrm{kg} / \mathrm{m}^{2}$
Any comorbidities
Diabetes
Hypertension
Cardiovascular disease
Chronic obstructive pulmonary disease
Malignancy
Chronic liver disease
Chronic kidney disease
Immunocompromised ${ }^{*}$
APACHE II
Time from illness onset to ICU admission, days
Medication use
Norepinephrine use
Maximum infusion rate in first $24 \mathrm{~h}$ on ICU,
Corticosteroids
Remdesivir
Anakinra

Symptoms and (laboratory) parameters

Heart rate, bpm
Mean arterial pressure, $\mathrm{mmHg}$
Fluid balance in first $24 \mathrm{~h}$ on ICU,
Urine output in first $24 \mathrm{~h}$ on ICU, $\mathrm{mL}$
Creatinine, $\mu \mathrm{mol} / \mathrm{L}$
Dialysis
Mechanical ventilation (invasive)
Tidal volume, $\mathrm{mL} / \mathrm{kg}$
Respiratory rate, bpm
$\mathrm{PEEP}, \mathrm{cm} \mathrm{H}_{2} \mathrm{O}$
FiO $2, \%$
P/F ratio
$100-200$
$200-300$
Thrombocytes, $10^{9} / \mathrm{L}$
Leukocytes, $10^{9} / \mathrm{L}$
C-reactive protein, $\mathrm{mg} / \mathrm{L}$
Procalcitonin, $\mu \mathrm{g} / \mathrm{L}$
Ferritin, $\mu \mathrm{g} / \mathrm{L}$

All patients $(\boldsymbol{n}=24)$
$69[61-73]$
$6(25 \%)$
$18(75 \%)$
$27.5[24.3-31.1]$
$19(79 \%)$
$7(29 \%)$
$6(25 \%)$
$7(29 \%)$
$3(13 \%)$
$10(42 \%)$
$0(0 \%)$
$1(4 \%)$
$5(17 \%)$
$17[11-21]$
$11[8-13]$

$20(83 \%)$

$0.11[0.07-0.21]$

$1(4 \%)$

$3(13 \%)$

$19(79 \%)$

$1(4 \%)$

$83[71-112]$

77 [72-81]

1348 [680-1881]

1105 [888-1486]

86 [70-133]

$0(0 \%)$

$24(100 \%)$

$5.3[4.4-6.0]$

21 [20-24]

12 [10-14]

50 [41-60]

164 [136-189]

$20(83 \%)$

$4(17 \%)$

239 [151-274]

$8.2[5.3-11.6]$

301 [157-316]

$0.72[0.29-3.66]$

1216 [488-1834]
Table 1 Patient characteristics (Continued)

\begin{tabular}{ll}
\hline Characteristics & All patients $(\boldsymbol{n}=24)$ \\
\hline Lactate (highest over last $24 \mathrm{~h}), \mathrm{mmol} / \mathrm{L}$ & $1.2[1.1-1.7]$ \\
D-dimer, $\mathrm{ng} / \mathrm{mL}$ & $3075[1780-4598]$ \\
Troponin I, ng/L & $23[13-44]$ \\
Albumin, g/L & $20[17-22]$ \\
Alanine aminotransferase, U/L & $34[21-41]$ \\
Aspartate aminotransferase, U/L & $48[31-73]$ \\
Creatinine kinase, U/L & $136[56-357]$ \\
Lactate dehydrogenase, U/L & $398[303-499]$ \\
Outcome parameters & \\
Secondary infections & $0(0 \%)$ \\
Death & $2(8 \%)$ \\
\hline
\end{tabular}

Data were obtained at study inclusion and are presented as $n(\%)$ or median [IQR]

${ }^{*}$ Chronic use of immunosuppressive medication

patient characteristics were obtained at admission to our ICU. Data were analysed using SPSS Statistics v22 (IBM, Armonk, USA) and GraphPad Prism v8.3.0 (GraphPad Software, La Jolla, USA).

Patient characteristics are listed in Table 1. In line with previous observations [3], the majority of patients was male and many had comorbidities. The median time from onset of COVID-19 symptoms to ICU admission was 11 days. All patients were mechanically ventilated and exhibited increases in inflammatory parameters (Table 1). As of March 27, 2020, two patients died (at 3 and 4 days post-ICU admission, data of only one timepoint of these patients was recorded), and 22 patients were still in the ICU.

Although mHLA-DR expression levels in COVID-19 patients were lower than those observed in healthy subjects $(15,000-45,000 \mathrm{mAb} / \mathrm{cell}$ [5]), the extent of suppression was less pronounced than observed in bacterial septic shock patients (geometric mean [95\% CI] of 11,860 [11,035-12,746] vs. 5211 [4904-5537] $\mathrm{mAb} /$ cell, respectively; $p<0.0001$; Fig. 1a, sepsis data from [1]). mHLA-DR expression kinetics revealed no change over time (Fig. 1b). Circulating C-reactive protein concentrations declined over time (Fig. 1c), whereas no significant changes in circulating procalcitonin, leukocytes, or ferritin levels were observed (Fig. 1d-f). None of the patients developed a secondary infection during the follow-up period (last recorded timepoint: 16-17 days post-ICU admission, see Fig. 1).

In conclusion, despite a pronounced inflammatory response in COVID-19 patients, our preliminary results indicate more moderate innate immune suppression compared with bacterial septic shock 

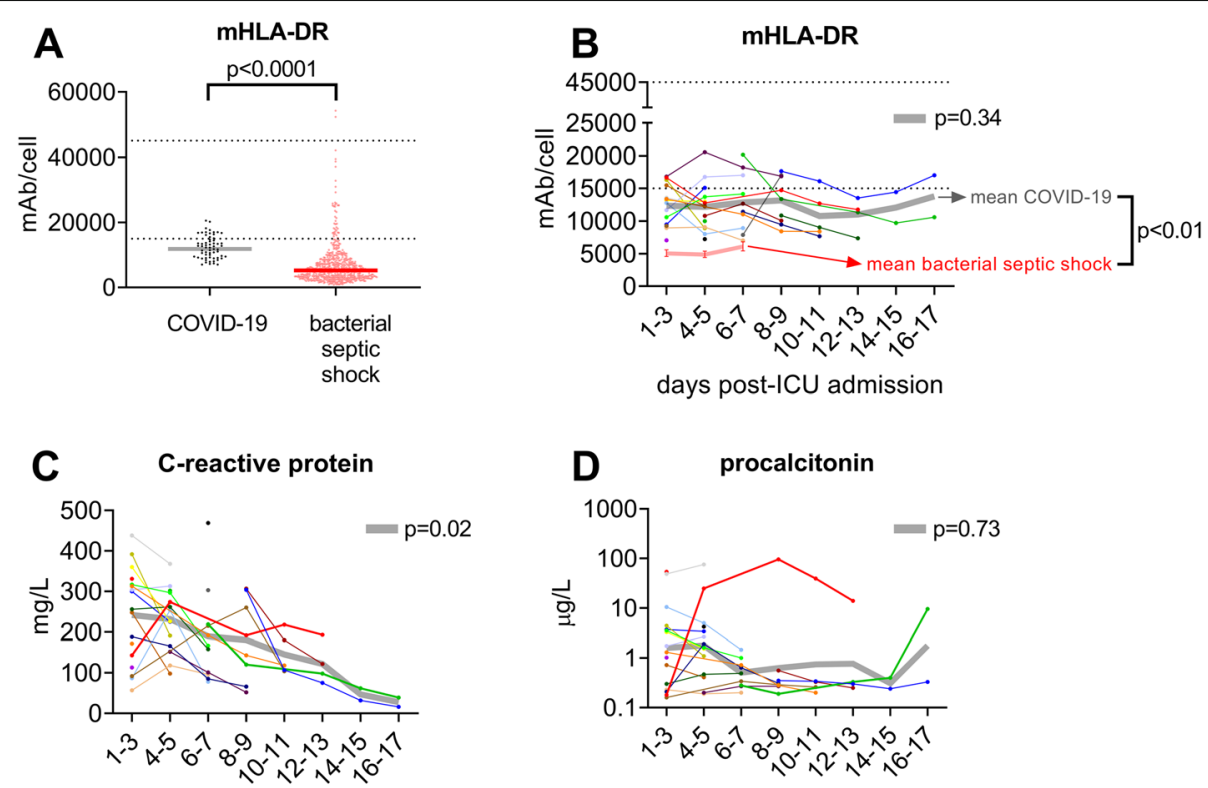

days post-ICU admission

days post-ICU admission

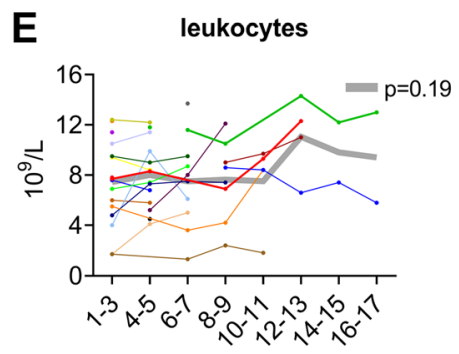

days post-ICU admission

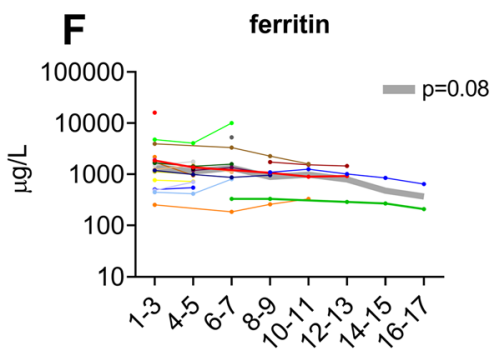

days post-ICU admission

Fig. 1 a mHLA-DR expression in patients with COVID-19 $(n=24$, multiple timepoints) and bacterial septic shock $(n=241$, days $1-2,3-4$, and/or $6-$ 8 after onset of septic shock, obtained using the same methodology, data recently published [1]). Horizontal line indicates geometric mean. The dotted lines indicate the reference range in healthy subjects [5]. $p$ value calculated using unpaired $t$ test on log-transformed data. $\mathbf{b}$-f Kinetics of mHLA-DR expression, circulating C-reactive protein, procalcitonin, leukocyte numbers, and ferritin in COVID-19 patients (individual data are shown, $n=24)$. The transparent grey line represents mean $(\mathbf{b}, \mathbf{c}, \mathbf{e})$ or geometric mean $(\mathbf{c}, \mathbf{e})$ values of the entire cohort. The transparent pink line in b represents data obtained from [1] (geometric mean $\pm 95 \%$ Cl, please note that values obtained at days 1-2 ( $n=203), 3-4(n=205)$, and 6-8 $(n=133)$ after onset of septic shock are plotted at days $1-3,4-5$, and $6-7$, respectively). The dotted lines in $\mathbf{b}$ indicate the reference range in healthy subjects [5]. $p$ values next to the transparent grey line represent changes over time in COVID-19 patients, calculated using mixed model analysis (on log-transformed data for $\mathbf{d}$ and $\mathbf{f}$ ). Differences between COVID-19 and sepsis patients in $\mathbf{b}$ were analysed using unpaired $t$ tests on log-transformed data ( $p<0.0001$ on days $1-3$ and $4-5$, and $p=0.0015$ on days $6-7$ )

patients. These findings are in accordance with a low incidence of secondary infections in COVID-19 patients. Therefore, innate immune suppression as a negative feedback mechanism following pathogenassociated molecular pattern-induced inflammation appears less pronounced in COVID-19.

\section{Acknowledgements}

Next to the authors of this letter, the RCI-COVID-19 study group consists of Pleun Hemelaar, Remi Beunders, Johannes van der Hoeven, Sjef van der Velde, Hetty van der Eng, Noortje Rovers, Margreet Klop-Riehl, Jelle Gerretsen, Emma Kooistra, Nicole Waalders, Wout Claassen, Hidde Heesakkers, Tirsa van Schaik, Mihai Netea, Leo Joosten, Nico Janssen, Inge Grondman, Aline de Nooijer, Quirijn de Mast, Martin Jaeger, Ilse Kouijzer, Helga Dijkstra, Heidi Lemmers, Reinout van Crevel, Josephine van de Maat, Gerine Nijman, Simone
Moorlag, Esther Taks, Priya Debisarun, Heiman Wertheim, Joost Hopman, Janette Rahamat-Langendoen, Chantal Bleeker-Rovers, Esther Fasse, Esther van Rijssen, Manon Kolkman, Bram van Cranenbroek, Ruben Smeets, and Irma Joosten. All of these authors are affiliated to the Radboud Center of Infectious Diseases.

\section{Authors' contributions}

MK and PP designed the study. TF, JS, and FvdV were responsible for the data collection. HK performed the flow cytometric analysis. MK performed the statistical analysis and drafted the manuscript. TF, JS, FvdV, HK, and PP critically revised the manuscript. All authors read and approved the final manuscript.

Funding

The work was internally funded by the participating departments. 


\section{Availability of data and materials}

All data generated or analysed during this study are included in this published article.

\section{Ethics approval and consent to participate}

The study was carried out in accordance with the applicable rules

concerning the review of research ethics committees and informed consent in the Netherlands. All patients or legal representatives were informed about the study details and could abstain from participation.

\section{Consent for publication}

Not applicable.

\section{Competing interests}

The authors declare that they have no competing interests.

\section{Author details}

'Department of Intensive Care Medicine, Radboud University Medical Center, Internal Mail 710, PO Box 9101, 6500 HB Nijmegen, The Netherlands.

${ }^{2}$ Radboud Center for Infectious Diseases (RCI), Radboud University Medical

Center, Nijmegen, The Netherlands. ${ }^{3}$ Department of Internal Medicine, Radboud university medical center, Nijmegen, The Netherlands. ${ }^{4}$ Department of Medical Immunology, Radboud university medical center, Nijmegen, The Netherlands.

Received: 6 April 2020 Accepted: 14 April 2020

Published online: 26 May 2020

\section{References}

1. Leijte GP, Rimmele T, Kox M, Bruse N, Monard C, Gossez M, Monneret G, Pickkers $P$, Venet F. Monocytic HLA-DR expression kinetics in septic shock patients with different pathogens, sites of infection and adverse outcomes. Crit Care. 2020;24(1):110.

2. Mehta P, McAuley DF, Brown M, Sanchez E, Tattersall RS, Manson JJ, Hlh Across Speciality Collaboration UK. COVID-19: consider cytokine storm syndromes and immunosuppression. Lancet. 2020;395(10229):1033-4.

3. Huang C, Wang Y, Li X, Ren L, Zhao J, Hu Y, Zhang L, Fan G, Xu J, Gu X, et al. Clinical features of patients infected with 2019 novel coronavirus in Wuhan, China. Lancet. 2020;395(10223):497-506.

4. Ruan Q, Yang K, Wang W, Jiang L, Song J. Clinical predictors of mortality due to COVID-19 based on an analysis of data of 150 patients from Wuhan, China. Intensive Care Med. 2020;46(5):846-8.

5. Remy S, Gossez M, Belot A, Hayman J, Portefaix A, Venet F, Javouhey E, Monneret G. Massive increase in monocyte HLA-DR expression can be used to discriminate between septic shock and hemophagocytic lymphohistiocytosis-induced shock. Crit Care. 2018;22(1):213.

\section{Publisher's Note}

Springer Nature remains neutral with regard to jurisdictional claims in published maps and institutional affiliations. 